\title{
Immediate Outcomes of Traumatic Brain Injury at a Tertiary Care Hospital Of Pakistan- A Retrospective Study
}

\author{
Maria Khan \\ Dow University Of Health Sciences \\ Uzair Yaqoob ( $\square$ ozair_91393@hotmail.com ) \\ Zair Hassan \\ Lady Reading Hospital \\ Muhammad Muizz Uddin \\ Shaheed Mohtarma Benazir Bhutto Medical College
}

Dow University of Health Sciences https://orcid.org/0000-0002-5910-2875

\section{Research}

Keywords: Traumatic brain injury, outcome, Glasgow coma score, trauma, road traffic accidents, head injury

Posted Date: October 2nd, 2020

DOI: https://doi.org/10.21203/rs.3.rs-84330/v1

License: @ (1) This work is licensed under a Creative Commons Attribution 4.0 International License. Read Full License 


\section{Abstract}

Background: Traumatic Brain Injury (TBI) which is the leading cause of morbidity and mortality all over the world and the impact is much worse in Pakistan. The objective of the study is to describe the epidemiological characteristics of patients with TBI in our country and to determine the immediate outcomes of patients with TBI after the presentation.

Method: This retrospective study was conducted at the Lady Reading Hospital. Data were extracted from the medical record room from January $1^{\text {st }}$ to December $31^{\text {st }}, 2019$. The severity of TBI was based on Glasgow Coma Scale (GCS) and was divided into mild (GCS 13-15), moderate (GCS 9-12), and severe TBI (GCS $\leq 8$ ) based on the GCS. SPSS v.23 was used for data analysis.

Results: Out of 5047 patients, 3689 (73.1\%) males and 1358 (26.9\%) females. The most commonly affected age group was 0-10 years $(25.6 \%)$ and $21-30$ years $(20.1 \%)$. was the predominant cause of injury $(38.8 \%, n=1960)$ followed by fall $(32.7 \%, n=1649)$. Most $(93.6 \%, n=4710)$ of the TBls were mild. After the full initial assessment and workup, and completing all first-aid management, the immediate outcome was divided into four, most frequent $(67.2 \%, n=3393)$ of which was "disposed (discharged)", and 9.3\% ( $n=470)$ were admitted for further management.

Conclusion: Our study represents a relatively conclusive picture of epidemiological data on the burden of TBI in Pakistan. Although a large proportion of patients had a mild TBI, they may likely be under-diagnosed. This warrants for further investigation of MTBI in population-based studies across the globe.

\section{Introduction:}

Traumatic injury is the leading cause of mortality among males and females aged 15 to 44 years. ${ }^{1}$ It leaves a devastating effect on the patient's life. Every year 1.2 million lives are lost to road traffic accidents (RTA), and approximately 50 million others are subjected to various forms of other injuries. ${ }^{2}$ Studies have shown that RTAs are particularly prevalent in low and middle-income countries. ${ }^{3}$ Pakistan, among several other east Mediterranean countries, has a significant contribution to the mortality rate from such accidents. ${ }^{4}$ Head injury is the leading cause of death and disability following traumatic injury. ${ }^{5}$ It accounts for 1.7 million cases in the United States (US) annually, particularly in people younger than 45 years of age. ${ }^{6}$ The phrase "Traumatic brain injury (TBI)" is now being used instead of "head injury", representing a disruption in brain function or any other evidence of pathology of the brain, secondary to an external force. The burden of TBI is highest in developing countries. The incidence of TBI is still rising, owing to the wider use of motor vehicles in these countries, whereas TBI secondary to falls is largely reported in high-income countries. ${ }^{7}$

The lack of epidemiological data on TBI in developing countries, particularly Asian countries reflects a gap in health information systems for Asia. Several studies have shown the variation in prognostic factors, outcomes, and laboratory results in patients with TBl; however, more studies should be conducted to focus on the risk factors, types, incidence, and global distribution of TBI. To date, there are very few researches and guidelines that cover all the essential elements of TBI. Not only it's a challenge for physicians to optimize the management of TBI, comprehending the significance of the epidemiology of TBI is equally important and essential for the public health sector to provide optimal medical and preventive strategies. Also of importance, many of the risk factors related to TBI are modifiable; hence, studies should be centered on preventive approaches taken by general practitioners and authorities responsible for the public health interest.

Our study aims to describe the epidemiological characteristics of patients with TBI in our country and to bridge the gap between epidemiological data concerning TBI from developed and developing regions across the globe.

\section{Materials And Methods:}

This retrospective study was conducted at the neurosurgery trauma unit of the Lady Reading Hospital, a tertiary care hospital of Peshawar, Pakistan, from January 1st to December 31st, 2019. Ethical approval was obtained from the institutional review board of Hayatabad medical complex, Peshawar. A total of 5047 patients with TBI were registered. All patients went through complete neurological examination including the Glasgow Coma Score (GCS) after giving first-aid treatment. The rest of the systemic 
examination was also performed to rule out any other major organ involvement. A complete history was taken from the family and/or eyewitnesses accompanying the patients including the cause of injury (RTA, fall, gunshot, and others). The patients were then referred to the radiological department for an emergency Computed Tomography (CT) scan.

The severity of TBI was divided into mild (GCS 13-15), moderate (GCS 9-12), and severe (GCS $\leq 8$ ) based on the GCS. Following the Canadian CT head rule, patients even with mild head injury were sent for the CT scan, and those with moderate and severe head injury were already the candidates for CT scan. ${ }^{8}$

Patients with a mild head injury and normal CT scan were discharged and those with evident abnormalities identified in the scans and/or severe head injury were admitted. Patients whose scans were doubtful to the on-call resident and those with moderate head injury were detained for a minimum of four hours for repeat clinical and radiological assessment. Those having a primary complaint other than that of the brain were referred to the appropriate department for evaluation. Patients leaving against medical advice were excluded.

Data entry and analysis was done on SPSS v.23. Frequency and percentages were calculated for age groups, gender, mode, and severity of the injury, and outcomes.

\section{Results:}

Over the one year study period, a total of 5047 patients were included in the study. An average of 13 patients per day was presented to the Accident and Emergency Department. Out of 5047 patients, 3689 (73.1\%) were males and 1358 (26.9\%) were females The mean age was $25.9 \pm 3.7$ years and most of them were less than 40 years of age (Table 1 ).

\section{Table 1: Presentation according to age groups}

\begin{tabular}{|l|l|l|}
\hline Age in years & Frequency & Percentage \\
\hline $0-10$ & 1292 & $25.6 \%$ \\
\hline $11-20$ & 817 & $16.2 \%$ \\
\hline $21-30$ & 1014 & $20.1 \%$ \\
\hline $31-40$ & 984 & $19.5 \%$ \\
\hline 41.50 & 429 & $8.5 \%$ \\
\hline 51.60 & 250 & $4.9 \%$ \\
\hline 61.70 & 200 & $3.9 \%$ \\
\hline 71.80 & 61 & $1.2 \%$ \\
\hline
\end{tabular}

Regarding the mode of injury, road traffic accident (RTA) was found to be the major cause of injury $(38.8 \%, n=1960)$, followed by fall $(32.7 \%, n=1649)$, as shown in table 2.

Table 2: Type of Trauma

\begin{tabular}{|l|l|l|}
\hline Type (daily average) & Frequency & Percentage \\
\hline RTA & 1960 & $38.8 \%$ \\
\hline Fall & 1649 & $32.7 \%$ \\
\hline Gunshot & 7 & $0.15 \%$ \\
\hline Other & 1431 & $28.4 \%$ \\
\hline
\end{tabular}

According to the GCS score, most $(93.6 \%, n=4710)$ of the TBIs were mild with a GCS of $13-15$ as shown in table 3.

Table 3: Severity of traumatic brain injury based on Glasgow coma score

\begin{tabular}{|l|l|l|}
\hline Severity (Glasgow coma score) & Frequency & Percentage \\
\hline Mild (13-15) & 4710 & $93.3 \%$ \\
\hline Moderate (8-12) & 231 & $4.6 \%$ \\
\hline Severe (3-7) & 108 & $2.1 \%$ \\
\hline
\end{tabular}

After the full initial assessment and workup, and completing all first-aid management, the immediate outcome was divided into four, most frequent $(67.2 \%, n=3393)$ of which was "disposed (discharged)", and 9.3\% ( $n=470)$ were admitted for further management (Table 4).

Table 4: Immediate outcome 


\begin{tabular}{|l|l|l|}
\hline Outcome (daily average) & Frequency & Percentage \\
\hline Admissions & 470 & $9.3 \%$ \\
\hline Disposed & 3393 & $67.2 \%$ \\
\hline Detained and Disposed & 826 & $16.4 \%$ \\
\hline Referred & 358 & $7.1 \%$ \\
\hline
\end{tabular}

\section{Discussion:}

Traumatic brain injury is the most important cause of death following multiple traumas. In many instances, severe damage to the brain was found to be the leading cause of early departure or fatality in the first week after a traumatic experience. ${ }^{9}$ The increasing incidence of traumatic brain injury is associated with several factors such as motor vehicle-related injuries, falls, and assaults, which are mostly comprised of gunshot wounds to the head and neck region. It has been well established that the leading cause of brain injury is motor vehicle-related injuries. The most severe manifestations of brain injury are often seen in road traffic accidents. ${ }^{10}$ The conventional classification of TBI is based on the mechanism of injury, severity, and structural damage. Similar to our study, clinical severity in TBI is assessed universally by the Glasgow coma score. Lastly, neuroimaging is a convenient tool for evaluating structural damage. ${ }^{11}$

TBI is broadly classified into primary and secondary types. Primary Injury occurs at the time of external impact brain and results in a concussion, contusion, laceration, or diffuse axonal injury. Secondary Injury takes place hours to days after the initial insult. It is comprised of complications due to primary injury such as systemic hypotension, hypoxia, or an increase in intracranial pressure leading to cerebral edema. A series of cellular and biochemical reactions resulting in mitochondrial damage, and cell death and necrosis form the basis of secondary injury. ${ }^{12,13}$ Importantly, hypotension and hypoxia fall under the most acute and easily treatable mechanisms of secondary injury. ${ }^{14}$ Hence, early preventive measures and treatment strategies are important in stabilizing the patient, so that secondary injury can be prevented. The current study found a mild TBI in $93.6 \%$ of patients with a GCS of $13-15$. Moreover, $67.2 \%$ of these patients were discharged just after first-aid management.

The statistics concerning TBI from low and middle-income countries are scarce. The epidemiology of TBI across various regions and socioeconomic divides was shown in a study by Dewan et al. in which the authors established a comprehensive relationship between RTA and TBI. Approximately 69 million cases of TBI emerge each year globally with the highest incidence in America and Canada, with a proportion of $29 \%$ of RTA that resulted in TBI compared to $34 \%$ in Southeast Asia and the pacific, reflecting a more significant overall burden in this region. ${ }^{15}$ This is on par with our data showing $38.8 \%$ of TBI were due to RTA. These findings are maybe attributable to the association between traffic regulations and the incidence of RTA across the globe. ${ }^{16}$ Moreover, the scarcity of sidewalks, traffic lights, and safety measures by pedestrians and cyclists contribute to TBI following RTA. An increasing level of motor vehicles in our country is proportional to RTA associated deaths. Additionally, the use of helmets and seatbelts is not common in Pakistan. ${ }^{17}$ In contrast, falls are a more frequent cause of TBI in elderly and children. ${ }^{18}$

Although the region-specific data from Asia is limited, the global burden of disease (GBD) study provides some insight into TBI related outcomes in India, China, and other Asian regions. Our research also shows that falls are the second most common cause of TBI. Compared to the other areas in GBD where RTA contributes to a majority of the TBI cases, falls are a leading cause of TBI in Asia whereas individual country data shows that RTA is the leading cause of TBI in India and China accounting for approximately 45$60 \%$ and $61 \%$ cases respectively. ${ }^{19}$ These results are consistent with our study. Despite the lack of literature on the epidemiology of TBI in Pakistan, Raja et al. conducted a survey in Pakistan to determine the demographics of head and spinal injuries from public sector hospitals. The annual incidence of TBI in Pakistan was estimated to be 50/100,000 of the total population. The study also addressed RTA as the most prevalent cause of TBI. ${ }^{20}$

Our study result shows that the majority of the patients were males (73\%) and was found to be in the first decade of life. Falls are the leading cause of TBI in this age group, particularly in children aged less than five years just like Saher M et al. reported. This could be as a result of parental negligence. ${ }^{17,21,22} \mathrm{~A}$ meta-analysis by Nguyen et al. also showed a higher incidence rate of TBI in males and for combined adolescents and adults. ${ }^{6}$ This trend could be a result of young adults and males, in particular, engaging in more risky and impulsive behavior such as reckless driving. ${ }^{23}$ Likewise, a compilation of Retrospective and prospective studies on the epidemiology of TBI in Europe reported a mean age of $26.7 .^{24}$ Similarly, the mean age calculated in our study was 25.9. A vast literature provides evidence that there was always a male predominance irrespective of age, severity, and mechanism of injury. ${ }^{10,12,18}$ 
Regarding hospital admissions surveys by the National Institutes of Health Consensus Development Panel on Rehabilitation of Persons with TBI show that TBI represents $15.1 \%$ of all hospital admissions in the USA. ${ }^{25}$ In contrast, this study reveals that only $9.9 \%$ of patients were admitted, whereas a vast majority of them were discharged. People with low income tend to underreport; this could be a possible explanation for the low hospitalization frequency among people who encounter head injury given the socioeconomic status of the majority of the population in our country. ${ }^{26}$ Likewise, a retrospective study of patients with TBI presenting to hospitals in California demonstrated that the majority of these patients (78.9\%) were discharged. This is in accordance with our results and highlights that most of the cases of TBI are mild and hence, do not require hospitalization. ${ }^{27}$

The literature search showed that mild TBI (MTBI) is more prevalent than moderate or severe. A systematic review by the world health organization showed that $70-90 \%$ of all treated brain injuries are mild. Moreover, adolescents in the age group of 16-20 years have a higher frequency of MTBI compared to other age groups. This again could be due to a more active and carefree attitude amongst them. Patients presenting with MTBI are usually not admitted to hospitals; therefore, such cases are likely to be overlooked. These results were similar to our study. Such patients can present with chronic symptoms later in life. Moreover, there is a high probability of MTBI to be under-diagnosed. As such, the need to call for better interventions by physicians plays an integral part in preventing long-lasting symptoms in these patients. ${ }^{6,28-30}$

There are several limitations in our study that need to be considered. Firstly, this was a single-center study with a small number of patients; hence, it does not cover TBI related incidents in other centers. Similarly, factors such as the mechanism of injury, rural vs. urban incidence, and occupation of patients were not taken into account. Moreover, we did not follow the patients until the end and assess their outcome. Secondly, we believe there may be that MTIB may have been underestimated or overlooked. Lastly, minor discrepancies in the definitions of TBI used by various researchers and conflicting severity scores may have influenced our results.

\section{Conclusion:}

Our study represents a relatively conclusive picture of epidemiological data on the burden of TBI in Pakistan. Although MTBI constitutes a large proportion of patients, they may likely be under-diagnosed. This warrants for further investigation of MTBI in population-based studies across the globe. Our study provides a valuable insight into the incident rate, demographics, and outcomes of TBI in our country. Given the financial burden of the existing pool of TBI management in our country, more reserved management of head injury in hospitals should be observed, resulting in cost-effective use of hospital resources. Importantly steps to improve public awareness about RTA related TBI must be taken in conjunction with other general measures for TBI care.

\section{Declarations:}

ETHICS APPROVAL AND CONSENT TO PARTICIPATE: The study was approved by the Institutional Review Board of Hayatabad medical complex, Peshawar, Pakistan with a waiver of the requirement of informed consent, since this is a retrospective study.

CONSENT FOR PUBLICATION: Not applicable

AVAILABILITY OF DATA AND MATERIALS: The datasets used and/or analyzed during the current study are available from the corresponding author on reasonable request.

\section{COMPETING INTERESTS: None}

\section{FUNDING: None}

AUTHORS' CONTRIBUTIONS: I, corresponding author of this manuscript, certify that I and my co-authors have contributed to all of the following:

1. Substantial contributions to conception and design, acquisition of data or analysis and interpretation of data.

2. Drafting the article or revising it critically.

3. Final approval of the version to be published.

4. Agreed to be accountable for all aspects of the work in ensuring that questions related to the accuracy or integrity of any part of the work are appropriately investigated and resolved. 


\section{References:}

1. Peden M, Scurfield R, Sleet D, Mohan D, Hyder AA, Jarawan E, et al. World report on road traffic injury prevention [Internet]. Geneva, Switzerland; 2004. Available from: https://apps.who.int/iris/bitstream/handle/10665/42871/9241562609.pdf? sequence $=1$.

2. Ahmadi A, Bazargan-Hejazi S, Heidari Zadie Z, Euasobhon P, Ketumarn P, Karbasfrushan A, et al. Pain management in trauma: A review study. J Inj Violence Res. 2016;8(2):89-98. doi:10.5249/jivr.v8i2.707.

3. Global status report on road safety 2013: supporting a decade of action [Internet]. Geneva, Switzerland: World Health Organization; 2013. Available from: https://www.who.int/violence_injury_prevention/road_safety_status/2013/report/Paho_Roadsafetymanual_english_051515.pdf.

4. Hyder AA, Razzak JA. The challenges of injuries and trauma in Pakistan: an opportunity for concerted action. Public Health. 2013;127(8):699-703. doi:10.1016/j.puhe.2012.12.020.

5. Murray CJ, Lopez AD. Global mortality, disability, and the contribution of risk factors: Global Burden of Disease Study. Lancet. 1997;349(9063):1436-42. doi:10.1016/S0140-6736(96)07495-8.

6. Nguyen R, Fiest KM, McChesney J, Kwon C-S, Jette N, Frolkis AD, et al. The International Incidence of Traumatic Brain Injury: A Systematic Review and Meta-Analysis. Can J Neurol Sci. 2016;43(6):774-85. doi:10.1017/cjn.2016.290.

7. Menon DK, Schwab K, Wright DW, Maas Al. Position statement: definition of traumatic brain injury. Arch Phys Med Rehabil. 2010;91(11):1637-40. doi:10.1016/j.apmr.2010.05.017.

8. Stiell IG, Wells GA, Vandemheen K, Clement C, Lesiuk H, Laupacis A, et al. The Canadian CT Head Rule for patients with minor head injury. Lancet. 2001;357(9266):1391-6. doi:10.1016/s0140-6736(00)04561-x.

9. Pfeifer R, Teuben M, Andruszkow H, Barkatali BM, Pape H-C. Mortality Patterns in Patients with Multiple Trauma: A Systematic Review of Autopsy Studies. PLoS One. 2016;11(2):e0148844. doi:10.1371/journal.pone.0148844.

10. Shaikh F, Waseem M. Head Trauma. In: StatPearls [Internet]. Treasure Island (FL): StatPearls Publishing; 2018. Available from: https://www.ncbi.nlm.nih.gov/books/NBK459169/.

11. Pushkarna A, Bhatoe HS, Sudambrekar SM. Head Injuries. Med journal Armed Forces India. 2010;66(4):321-4. doi:10.1016/S0377-1237(10)80008-5.

12. Pervez M, Kitagawa RS, Chang TR. Definition of Traumatic Brain Injury, Neurosurgery, Trauma Orthopedics, Neuroimaging, Psychology, and Psychiatry in Mild Traumatic Brain Injury. Neuroimaging Clin N Am. 2018;28(1):1-13. doi:10.1016/j.nic.2017.09.010.

13. Galgano M, Toshkezi G, Qiu X, Russell T, Chin L, Zhao L-R. Traumatic Brain Injury: Current Treatment Strategies and Future Endeavors. Cell Transplant. 2017;26(7):1118-30. doi:10.1177/0963689717714102.

14. Arlinghaus K, Shoaib A, Price T, editors. Neuropsychiatric assessment. In: Textbook of Traumatic Brain Injury. Washington, DC: American Psychiatric Association; 2005. pp. 63-5.

15. Dewan MC, Rattani A, Gupta S, Baticulon RE, Hung Y-C, Punchak M, et al. Estimating the global incidence of traumatic brain injury. J Neurosurg 2018;1-18. doi:10.3171/2017.10.JNS17352.

16. Chiu W-T, Huang S-J, Tsai S-H, Lin J-W, Tsai M-D, Lin T-J, et al. The impact of time, legislation, and geography on the epidemiology of traumatic brain injury. J Clin Neurosci. 2007;14(10):930-5. doi:10.1016/j.jocn.2006.08.004.

17. Umerani MS, Abbas A, Sharif S. Traumatic brain injuries: experience from a tertiary care centre in Pakistan. Turk Neurosurg. 2014;24(1):19-24. doi:10.5137/1019-5149.JTN.7080-12.1.

18. Peeters W, van den Brande R, Polinder S, Brazinova A, Steyerberg EW, Lingsma HF, et al. Epidemiology of traumatic brain injury in Europe. Acta Neurochir (Wien). 2015;157(10):1683-96. doi:10.1007/s00701-015-2512-7.

19. Puvanachandra P, Hyder A. The burden of traumatic brain injury in Asia: a call for research. Pak J Neurol Sci. 2009;4(1):27-32.

20. Raja IA, Vohra AH, Ahmed M. Neurotrauma in Pakistan. World J Surg. 2001;25(9):1230-7. doi:10.1007/s00268-001-0087-3.

21. Thurman DJ. The Epidemiology of Traumatic Brain Injury in Children and Youths: A Review of Research Since 1990. J Child Neurol. 2016;31(1):20-7. doi:10.1177/0883073814544363. 
22. Araki T, Yokota H, Morita A. Pediatric Traumatic Brain Injury: Characteristic Features, Diagnosis, and Management. Neurol Med Chir (Tokyo). 2017;57(2):82-93. doi:10.2176/nmc.ra.2016-0191.

23. Steinberg L. A Social Neuroscience Perspective on Adolescent Risk-Taking. Dev Rev. 2008;28(1):78-106. doi:10.1016/j.dr.2007.08.002.

24. Brazinova A, Rehorcikova V, Taylor MS, Buckova V, Majdan M, Psota M, et al. Epidemiology of Traumatic Brain Injury in Europe: A Living Systematic Review. J Neurotrauma. 2018. doi:10.1089/neu.2015.4126.

25. Gardner AJ, Zafonte R. Neuroepidemiology of traumatic brain injury. Handb Clin Neurol. 2016;138:207-23. doi:10.1016/B978-012-802973-2.00012-4.

26. Fife D. Head injury with and without hospital admission: comparisons of incidence and short-term disability. Am J Public Health. 1987;77(7):810-2. doi:10.2105/ajph.77.7.810.

27. Hsia RY, Markowitz AJ, Lin F, Guo J, Madhok DY, Manley GT. Ten-year trends in traumatic brain injury: a retrospective cohort study of California emergency department and hospital revisits and readmissions. BMJ Open. 2018;8(12):e022297. doi:10.1136/bmjopen-2018-022297.

28. Rickels E, von Wild K, Wenzlaff P. Head injury in Germany: A population-based prospective study on epidemiology, causes, treatment and outcome of all degrees of head-injury severity in two distinct areas. Brain Inj. 2010;24(12):1491-504. doi:10.3109/02699052.2010.498006.

29. El-Menyar A, Mekkodathil A, Al-Thani H, Consunji R, Latifi R. Incidence. Demographics, and Outcome of Traumatic Brain Injury in The Middle East: A Systematic Review. World Neurosurg. 2017;107:6-21. doi:10.1016/j.wneu.2017.07.070.

30. Skandsen T, Nilsen TL, Einarsen C, Normann I, McDonagh D, Haberg AK, et al. Incidence of Mild Traumatic Brain Injury: A Prospective Hospital, Emergency Room and General Practitioner-Based Study. Front Neurol. 2019;10:638. doi:10.3389/fneur.2019.00638. 